\title{
Function-Splitting Heuristics for Discovery of Microservices in Enterprise Systems
}

\author{
Adambarage Anuruddha Chathuranga De Alwis ${ }^{1}$, Alistair Barros ${ }^{1}$, \\ Artem Polyvyanyy ${ }^{2}$, and Colin Fidge ${ }^{1}$ \\ 1 Queensland University of Technology, Brisbane, Australia \\ \{adambarage.dealwis, alistair.barros, c.fidge\}@qut.edu.au \\ 2 The University of Melbourne, Parkville, VIC, 3010, Australia \\ artem.polyvyanyy@unimelb.edu.au
}

\begin{abstract}
We present heuristics that help to identify suitable consumer-oriented parts of enterprise systems which could be re-engineered as microservices. Our approach assesses the key structural and behavioural properties common to both enterprise and microservice systems, as needed to guide a microservices discovery process and coherently assess restructuring recommendations. Building upon existing business object and system structural definitions, we present heuristics for two fundamental areas of microservice discovery, namely function splitting based on object subtypes (i.e., the lowest granularity of software based on structural properties) and functional splitting based on common execution fragments across software (i.e., the lowest granularity of software based on behavioural properties). A prototype analysis tool was developed based on the defined heuristics and experiments show that it can identify microservice designs which support multiple microservice characteristics, such as high cohesion, low coupling, high scalability, high availability, and processing efficiency while preserving coherent features of enterprise systems. In particular, we illustrate the usefulness of this new approach by conducting a case study based on customer management systems: SugarCRM and ChurchCRM.
\end{abstract}

Keywords: Microservice discovery, system reengineering, cloud migration.

\section{Introduction}

Microservices have emerged as the latest style of service-based software allowing systems to be distributed through the cloud as fine-grained components, typically with individual operations, in contrast to services under a Service-Oriented Architecture (SOA) which include all logically related operations [1]. As such, microservices allow specific parts of systems and the business processes they support, down to individual tasks, to be scaled up and replicated through the cloud, and be flexibly composed in Web, mobile computing, and Internet-of-Things (IoT) applications. These benefits originally led Netflix, and now Twitter, eBay, Amazon and other Internet players, to develop novel architectures for software solutions as microservices. Nonetheless, microservices have so far not been adopted for the dominant form of software in businesses, namely enterprise systems, limiting such systems' evolution and their exploitation of the full benefits of cloud-enabled platforms such as Google Cloud, Amazon AWS and IoT [2]. 
Enterprise systems, such as enterprise resource planning (ERP), customer relationship management (CRM) and supply chain management are large and complex, and contain complex business processes encoded in application logic managing business objects (BOs), in typically many-to-many relationships [3]. Restructuring enterprise systems as microservices is technically cumbersome, requiring tedious search and identification of suitable parts of the system to restructure, program code rewrites, and integration of the newly developed microservices with the 'backend' enterprise systems. This is a costly and error-prone task for developers, because enterprise systems have millions of lines of code and thousands of BOs they manage, entailing a multitude of functional dependencies, in and across many software packages and modules. In addition, microservices are the most fine-grained and loosely-coupled form of software components upon which to restructure large-scale enterprise systems. This leads to major uncertainties about the best way to split enterprise systems functions as microservices, to achieve high scalability and availability and low system latencies through the cloud, while attaining high cohesion and low coupling between software components.

Automated software re-engineering techniques have been proposed to improve the efficiency of transforming legacy applications, addressing specifically cohesion and coupling of software packages and components using static analysis techniques that focus on source code and dynamic analysis techniques that focus on software execution recorded in system logs. Even though these analyses proposed to improve software search and metrics, studies show that the success rate of software re-modularisation techniques, especially for large systems, remains low [4]. The key stumbling blocks are the limited insights available from syntactic structures of software code for profiling software dependencies and not identifying the semantics available through the business object relationships [5].

Enterprise systems can provide enriched semantic insights, available through the BOs that they manage which influence the software structure and the processes they support. For instance, an order-to-cash process in SAP ERP is supported through functions of software components: multiple sales orders, deliveries shared across different customers, shared containers in transportation carriers, and multiple invoices and payments. To support this process, multiple functions are invoked asynchronously, reflecting BO relationship types and cardinalities, and are seen through cross-service interactions, correlations, and data payloads [6]. Such insights provided by BO relationships are promising for improving the feasibility of automated discovery applications. As examples, Pẽrez-Castillo et al. [7] used transitive closures of strong BO dependencies derived from databases to recommend software functions hierarchies, while Lu et al. [8] demonstrated process discovery using SAP ERP logs based on BOs.

This paper presents discovery techniques that help to identify suitable consumeroriented parts of enterprise systems which could be re-engineered as microservices with desired characteristics such as high cohesion, low coupling, high scalability, high availability and high processing efficiency. It does so by providing an abstraction of the systems architecture, using key structural and behavioural properties common to both enterprise and microservices systems, considered essential to guide microservices discovery processes and coherently assess their potential restructuring. The structural properties address the functional composition of software, namely functions and their BO 
Create-Read-Update-Delete (CRUD) operations, while behavioural properties focus on system executions, at the level of operation invocations, reflecting single-entry-singleexit sequences characteristic of these systems. This, in principle, allows enterprise systems to be analysed at different units of structural and behavioural granularity, and the resulting restructure recommendations to be conveniently assessed for preservation of structural and behavioural properties. This paper addresses two fundamental areas of microservice discovery, namely function-splitting based on object subtypes (i.e., the lowest granularity of software based on structural properties) and functional splitting based on common execution fragments across software (i.e., the lowest granularity of software based on behavioural properties). This, we argue, provides a solid basis for future development of further microservices discovery heuristics.

The remainder of the paper is structured as follows. Section 2 presents structural and behavioural properties of software systems, while Section 3 exploits these properties to propose heuristics for discovering microservises in enterprise systems. Section 4 discusses an implementation and validation of the proposed heuristics. Related work is summarized in Section 5. The paper closes with a conclusion.

\section{Structural and Behavioural Properties of Enterprise and Microservice Systems}

This section describes the essential properties of a target system architecture that comprise an enterprise system (ES) and a microservice (MS) system, which is depicted in Fig. 1. This architecture will be used in our MS discovery approach (detailed in Section 3). The architecture reflects a unified software structure for both an ES and MS system, since a proper system migration from an ES to MS system is an incremental process in which the most prominent components are extracted and remodularized as MSs first [1]. Such remodularized MSs run in a cloud setting and are integrated with the 'backend' enterprise system as depicted in the Fig. 1.

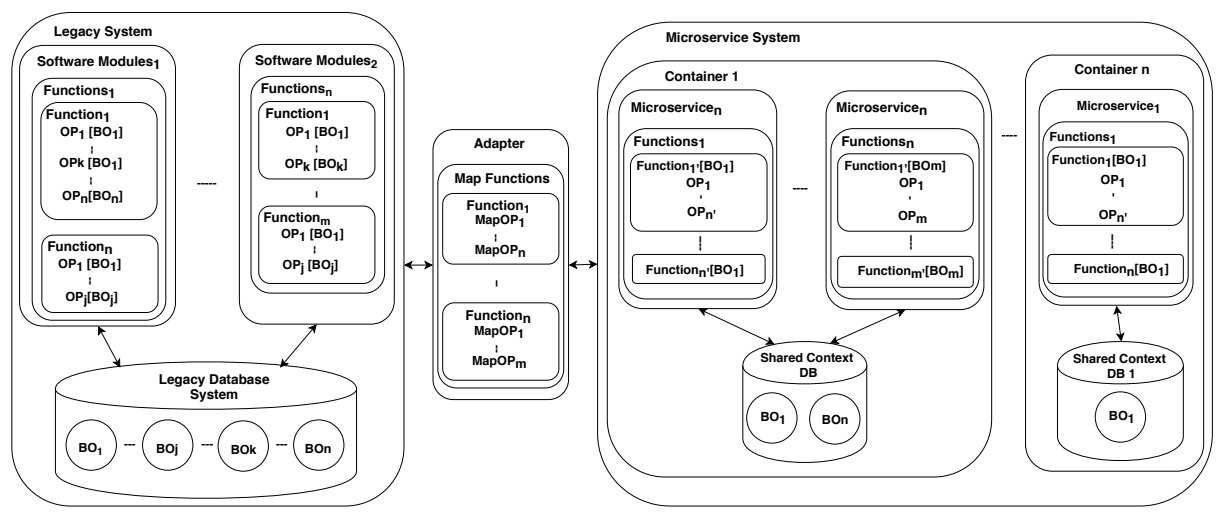

Fig. 1: Architecture of enterprise and microservices systems. 
The software structure of an ES (e.g., an ERP system) consists of a set of selfcontained modules (e.g., software components) drawn from different subsystems (e.g., production management), deployed on a specific execution platform. Modules consist of a set of functions (e.g., software classes) and each function consists of a number of operations (e.g., methods) aimed at manipulating BOs through CRUD operations which typically have database access logic or data processing logic applied to data stored in program variables and constants. The data stored in a centralized database associated with a deployed ES relates to BOs which process data resulting from business process executions supported through functions (such as transactions).

MSs are remodularized and potentially extended parts or functions of ESs, supporting consumer applications running in cloud applications. Since MSs are functionally isolated and loosely-coupled parts connected to each other, much like the components of a distributed system, they tend to concern individual BOs, locally managed through a database. The managed data of MSs is synchronized at discrete intervals with similar MS instances and with the backend ES. One or more MSs can run in an execution environment known as a cloud container, configured for specific execution characteristics, such as scalability or availability applying to all the MSs of the container.

Despite structural differences, the behaviour of an ES and an MS system is based on the invocation of operations, in well-defined processing sequences reflecting the relationships of BOs they manipulate. For example, the creation of a 'purchase order' will result in the invocation of functions involving the creation of 'line items' reflecting a strict containment of objects. Similarly, processing sequences between 'Shipper' and 'Shipping order' reflect weak containment while processing sequences between 'leads' and 'campaigns' reflect an association. In addition, normalization of a BO can result in additional process sequences. For example, the creation of a 'shipment' BO will result in an invocation of a function related to different shipments subtypes such as 'ground home delivery shipment' and 'intra UAESO shipment' based on the operational parameters provided at run time. These different execution sequences of operations reflect a set of single-entry-single-exit (SESE) regions [9] in an ES's executions.

Although an MS system executes in a manner similar to an ES, there are specific characteristics applicable only to an MS. Generally MSs run as distributed systems which are deployed through different containers that help the MS system to achieve high scalability and availability while executing services in an asynchronous manner and managing security through configurations of API gateways [1]. Scalability can be defined as allocation and de-allocation of the resources to containers on demand according to the configuration properties. Such configuration properties include load balancing and resource allocation policies optimizing the resources within a container allowing it to provide high scalability at lower cost. Furthermore, the configuration properties define circuit breaker threshold values for each MS which resides in a container, which assures that a request is redirected to another MS if it did not get a response from the initially accessed MS within the threshold time period resulting in high availability. Since there are multiple MSs in a single container, it can process multiple client requests in an asynchronous manner while aligning with the system requirements of the ES. All the MSs communicate through adapters which synchronize with the database system which resides in the legacy ES and this helps to achieve consistency among all the 
microservices which are distributed among multiple containers. Finally, each MS is developed in order to provide a specific functionality to the end user or the system, which makes them highly cohesive and loosely coupled services. This understanding leads us to the following formal characterization of the environment.

Let $\mathbb{I}$ and $\mathbb{O}$ be a universe of input types and output types, respectively. Let $\mathbb{O P}, \mathbb{T}$ and $\mathbb{B}$ be, respectively, a universe of operations, database tables, and business objects. Finally, let $\beta$ be a binary relation on $\mathbb{B}$ such that $\beta^{+}$is irreflexive ${ }^{3}$. Relation $\beta$ defines a subtype relation on business objects, i.e., for every $\left(b_{1}, b_{2}\right) \in \beta^{+}$we say that $b_{2}$ is a subtype of $b_{1}$. As proposed in this paper, techniques for the discovery of microservices rely on abstractions of ESs, as defined below.

The data related BOs in ESs are disseminated through several database tables.

Definition 2.1 (Business object).

A business object $b$ is characterized by a collection of database tables, i.e., $b \subseteq \mathbb{T}$. $\lrcorner$

The BOs in ESs have complex relationships with the operations which perform CRUD processes on them. Such operations are encapsulated in different functions of ESs and MSs.

Definition 2.2 (Operation).

An operation op is a triple $(I, O, T)$, where $I \in \mathbb{I}^{*}$ is a sequence of inputs, $O \in \mathbb{O}^{*}$ is a sequence of outputs, and $T \subseteq \mathbb{T}$ is a set of database tables. ${ }^{4}$

An ES can be seen as a finite automaton with operations as labels.

Definition 2.3 (Enterprise system).

An enterprise system is a 5 -tuple $\left(Q, \Lambda, \delta, q_{0}, A\right)$, where:

- $Q$ is a finite nonempty set of states,

$\circ \Lambda$ is a set of operations, such that $Q$ and $\Lambda$ are disjoint,

$\circ \delta: Q \times(\Lambda \cup\{\tau\}) \rightarrow \mathcal{P}(Q)$ is the transition function, where $\tau$ a is a special silent operation such that $\tau \notin Q \cup \Lambda$,

$\circ q_{0} \in Q$ is the start state, and

- $A \subseteq Q$ is the set of accept states. ${ }^{5}$

Let $\mathbb{C}$ and $\mathbb{M}$ be a universe of containers and microservices, respectively. Let $S$ be an enterprise system. By $\operatorname{SESE}(S)$, we denote the set of all (generalized) SESE fragments of $S$, cf. [9]; clearly, one can interpret an ES as a workflow graph with vertices defined by its states and a flow relation defined by its transition function. Each SESE fragment of an ES induces a function, or a call graph, i.e., a subgraph of ES. We abstract a function as a triple $(I, O, O P)$, where $I$ and $O$ are sequences of inputs and outputs, respectively, and $O P$ is a set of operations. For our purposes, we define a MSs system as follows.

Definition 2.4 (Microservices system). A microservices system of an enterprise system $S=\left(Q, \Lambda, \delta, q_{0}, A\right)$ is a 5-tuple $(S, C, M, \sigma, \mu)$, where:

${ }^{3}$ Given a binary relation $\alpha$, by $\alpha^{+}$we denote the transitive closure of $\alpha$.

${ }^{4}$ Given a set $A$, by $A^{*}$ we denote the set of all finite sequences that can be generated by concatenating elements of $A$.

${ }^{5}$ Given a set $A$, by $\mathcal{P}(A)$, we denote the powerset of $A$. 
- $C \subseteq \mathbb{C}$ is a set of containers,

- $M \subseteq \mathbb{M}$ is a set of microservices,

○ $\sigma: C \rightarrow \mathcal{P}(M) \backslash \emptyset$ is a deployment function that maps each container $c \in C$ onto a non-empty set of microservices $\sigma(c)$ that are deployed on $c$, and

- $\mu: M \rightarrow \mathcal{P}(\operatorname{SESE}(S)) \backslash \emptyset$ is a microservice definition function that maps each microservice $m \in M$ onto a non-empty set of SESE fragments, a.k.a functions, $\mu(m)$ of $S$, such that:

- No two microservices are defined using the same function, i.e., $\forall m_{1} \in M \forall m_{2} \in M:\left(m_{1} \neq m_{2}\right) \Rightarrow\left(\left(\mu\left(m_{1}\right) \cap \mu\left(m_{2}\right)\right)=\emptyset\right)$, and

- Every two functions used to define the microservices in $M$ are either disjoint, i.e., do not share an edge, or are in a subgraph relation.

Given an enterprise system $S,(S,\{c\},\{m\},\{(c,\{m\})\},\{(m,\{S\})\})$, where $c \in \mathbb{C}$ and $m \in \mathbb{M}$, is its elementary microservices system, or the elementary enterprise and microservices architecture induced by $S$.

\section{Automated Microservice Discovery}

As described in Section 2, the behaviour of an ES and an MS system is based on the invocation of functions which consist of well-defined sequences of operations governed by BO relationships. Such sequences illustrate a particular execution pattern based on the structure and behaviour of an organization. Therefore, we argue that a proper analysis of these sequences of operations will help to derive prominent microserviceable components. This assumption leads us to two heuristics which assist in MS discovery.

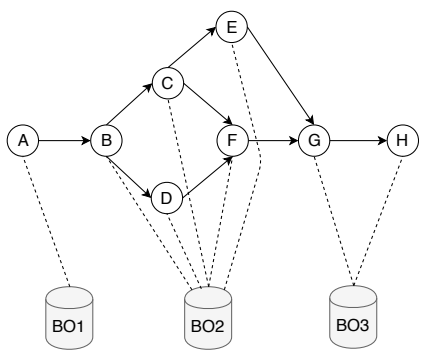

(a) Graph 1

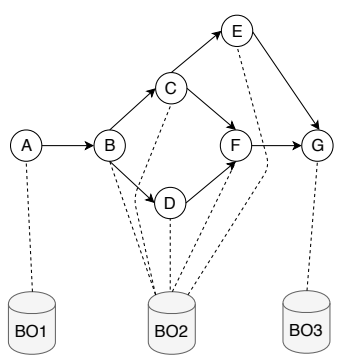

(b) Graph 2

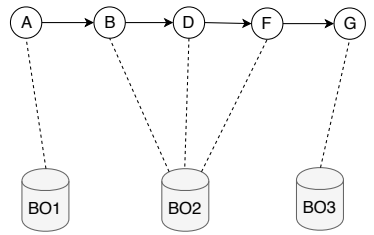

(c) Graph 3

Fig. 2: Patterns of system executions and BO relationships.

As an example, assume that an ES has three hypothetical processing sequences, as depicted in Fig. 2, in which each node of the sequences represents a system state after performing a CRUD operation. These states are linked to the BOs on which different CRUD operations were performed. Figs. 2(a) and 2(b) capture the same execution order dependencies between states 'A', 'B', 'C', 'D', 'E', 'F', and 'G'. Furthermore, the overlap between the execution patterns is high, i.e., more than $80 \%$, which emphasizes that CRUD operations were performed on the same BOs. For instance, the campaign 
management module in SugarCRM describes different types of publicity campaigns, such as newsletter, email, and non-email. The execution paths and the BOs they execute upon are similar. However, the BO attributes they use in the execution processes are often different. This execution behaviour is explicit because of the structural splitting of objects at the BO level, as described by Halpin and Morgan [10]. To address this phenomenon, we define Heuristic 1.

Heuristic 1. (Subtype) Given an enterprise system $S$, a subtype relation exists between a parent call graph $x=(I, O, O P) \in S E S E(S)$ and a child call graph $x^{\prime}=\left(I^{\prime}, O^{\prime}, O P^{\prime}\right) \in$ $\operatorname{SESE}(S)$, iff $I^{\prime} \subseteq I, O P^{\prime} \subseteq O P$, and $B^{\prime} \subseteq B$, where $B^{\prime}$ and $B$ are the BOs manipulated by $O P^{\prime}$ and $O P$, respectively. To ensure that the call graphs execute on the same BOs, we require that $80 \%$ of the states of the parent appear in the child.

In addition, some execution sequences can occur often when executing a software system. As an example, the execution pattern 'A', 'B', 'D', 'F', 'G' occurs in Figs. 2(a), 2(b), and 2(c). This phenomenon depends on the functional relationships that occur during execution time. For example, 'B' precedes ' $D$ ' in every execution because, for instance, the data in ' $\mathrm{B}$ ' is required for the execution of ' $\mathrm{D}$ '. In the functional structure level this can be described as a 'has a relationship' property, in which a class object of ' $D$ ' is referenced inside ' $B$ '. Such functional structure emphasizes that the same behaviour should be preserved in all the system executions. To address this issue, we define Heuristic 2.

Heuristic 2. (Common subgraph) Given an enterprise system $S$, a common subgraph of two call graphs $x, x^{\prime} \in \operatorname{SESE}(S)$ is a call graph $x^{\prime \prime} \in \operatorname{SESE}(S)$, such that $x^{\prime \prime} \subseteq x$ and $x^{\prime \prime} \subseteq x^{\prime}$

A common subgraph which captures frequent executions can be used as a basis for defining a microservice. This heuristic can be generalized to subgraphs common to multiple call graphs. Intuitively, choosing smaller common subgraphs produces smaller microservices which helps to achieve higher scalability. On the other hand, choosing larger subgraphs produces larger microservices which helps reduce communication overheads and improve system efficiency.

Heuristics 1 and 2 can guide the discovery of microservices that potentially support multiple microservice characteristics, such as high cohesion, low coupling, high scalability, availability, and processing efficiency, while preserving coherent features of enterprise systems. In what follows, this claim gets verified.

\subsection{Discovery Process}

Our microservice discovery and recommendation process based upon the above heuristics consists of two components, i.e., a Business Object Analyser (BOA) and a System Dynamic Analyser (SDA), as depicted in Fig. 3.

Since MSs are focused around accessing and transferring states of BOs, or partitions of BOs, in the system [11], it is important to identify the BOs in a given ES. Therefore, the BOA is comprised of a System Operation Extraction Model (SOEM) and a Business Object Derivation Model (BODM). The SOEM evaluates all the SQL queries to identify 


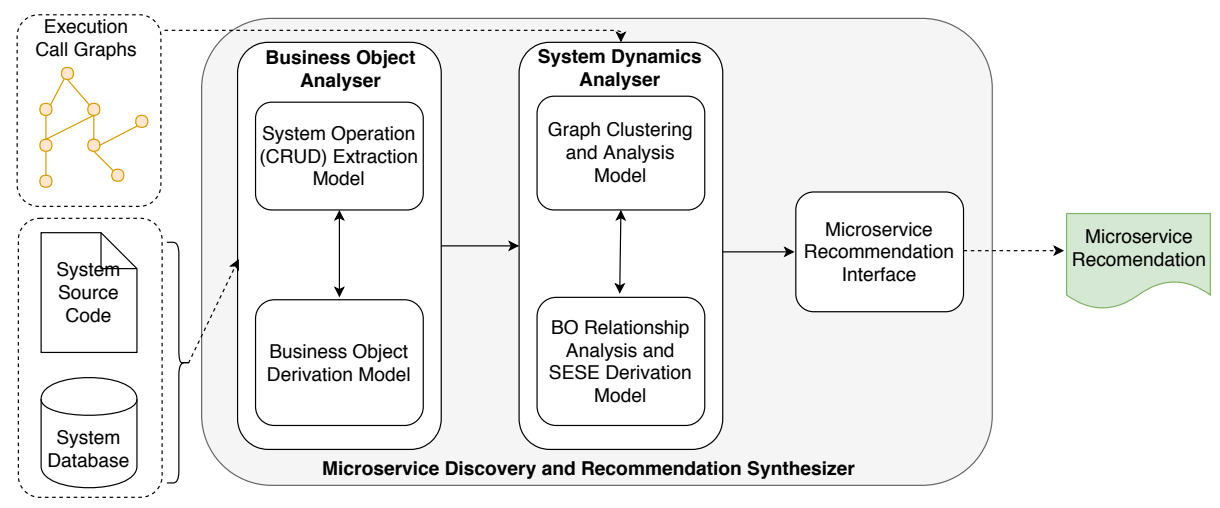

Fig. 3: An overview of our microservice discovery and recommendation process.

the relationships between database tables, while the BODM derives the BOs based on the identified relationships and data similarities, as described by Nooijen et al. [12].

The BOs identified by the BOA are provided as input to the SDA along with call graphs of the ES. The graph clustering and analysis model in the SDA identifies the Frequent Execution Patterns (FEP) in the provided set of $\operatorname{SESE}(S)$. These FEPs get evaluated against the aforementioned heuristics and classified into different categories, as described in Section 3.2. The categorized patterns are evaluated by BO relationship analysis and a SESE derivation model. The SESE derivation model identifies the BOs which are related to each node in the extracted graph pattern and the SESE regions related to each BO. Finally, the Microservice Recommendation Interface (MRI), provides different configuration models for MSs by evaluating the results of the system dynamic analysis model. Due to space limits, this paper only addresses the SDA and the MRI, which analyse the system execution patterns and recommend MS configuration models.

\subsection{Microservice Discovery Algorithms}

Given a set of call graphs of a legacy system, like the ones shown in Fig. 2, the SDA and MRI derive sets of MS recommendations based on Heuristics 1 and 2 using Algorithms 1 and 2. Algorithm 1 derives a set of subgraphs in the given set of call graphs of an ES, while Algorithm 2 analyses the subgraphs to identify functions which operate on single BOs to provide MS migration recommendations.

Algorithm 1 comprises four steps. The first step involves function GRAPHSUMMARY, which computes the set of adjacency matrices $M T$ of the call graphs $\operatorname{SESE}(S)$ (line 1). Each adjacency matrix is generated in two steps. First, the function constructs the set of all distinct states of the graphs. For example, for the three call graphs in Fig. 2, this set comprises states 'A', 'B', 'C', 'D', 'E', 'F', 'G', and 'H'. Then, for each call graph, the function creates a matrix $m t \in M T$ of size $N \times N$, where $N$ is the number of distinct states (for the graphs in Fig. 2, the number of distinct states is eight). In a matrix $m t \in M T$, a transition between two states $q$ and $q^{\prime}$ of the corresponding call graph is represented by ' 1 ' and the absence of a transition is represented by ' 0 '. 


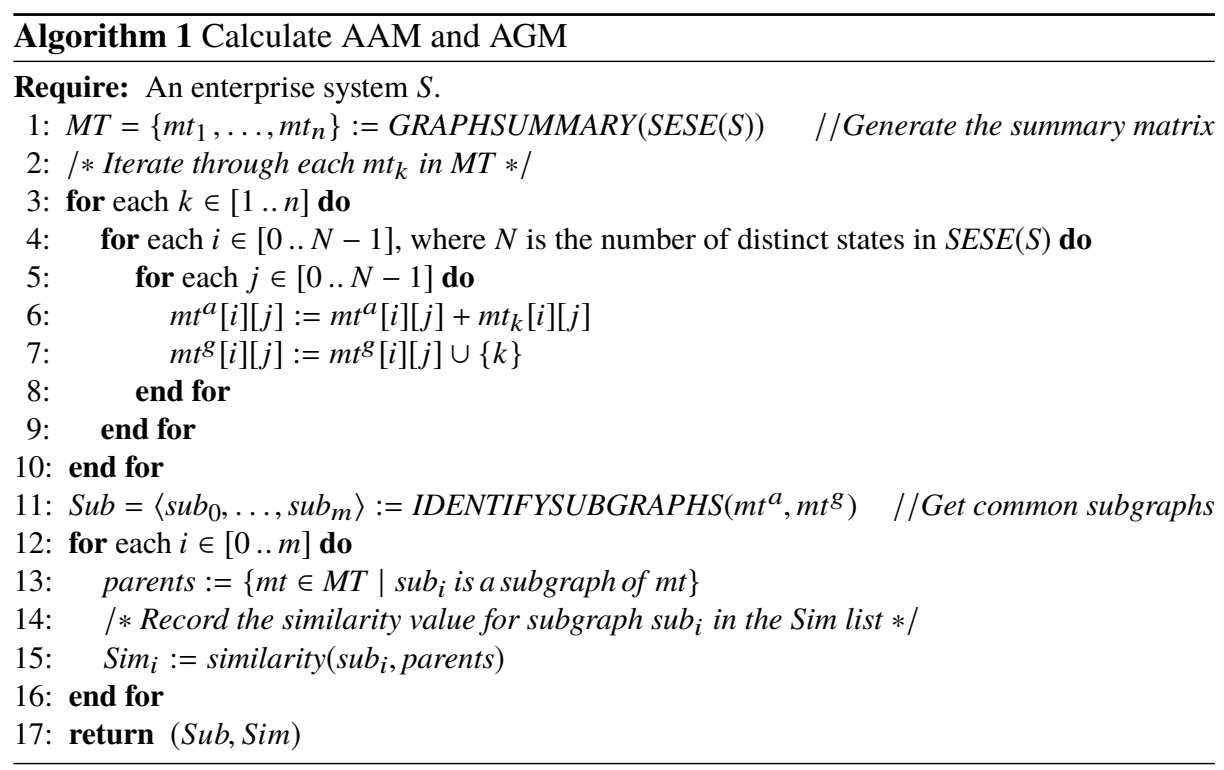

The second step of Algorithm 1 constructs two matrices, the Augmented Adjacency Matrix (AAM) $m t^{a}$ and Augmented Graph Matrix (AGM) $m t^{g}$, which are of size $N \times N$ (lines 3-10). At the beginning, all the values of $m t^{a}$ are initialized to ' 0 ' and all the values of $m t^{g}$ are initialized to the empty set. Then, the algorithm iterates over adjacency matrices $m t_{k} \in M T$ to compute statistics on transitions. The indices of the graphs that contain a transition are recorded in matrix $m t^{g}$ and the number of graphs that contain the transition gets stored in matrix $m t^{a}$. The AAM and AGM generated for the call graphs in Fig. 2 are shown in Fig. 4. In Fig. 4(a), the value in the AAM row 'A', column 'B' is ' 3 ' because all the three call graphs depicted in Fig. 2 have a transition (an edge) from node 'A' to node 'B'. Similarly, in Fig. 4(b), the value in the AGM row 'A', column 'B' encodes the graphs that contain the corresponding transition. Since the transition is in all the three call graphs, the value has been set to ' 1 ', '2', ' 3 '.

In the third step of Algorithm 1 the generated matrices $m t^{a}$ and $m t^{g}$ are passed as input to the IDENTIFYSUBGRAPHS function which computes the adjacency matrices of the common subgraphs $S u b$ of the call graphs (line 11).

In the fourth step, the algorithm iterates over subgraphs in $S u b$ and measures the similarity $\operatorname{Sim}_{i}$ between subgraph $s u b_{i}$ and all its parent graphs (lines 12-16). The similarity is identified as the ratio of the number of nodes in $s u b_{i}$ to the number of distinct nodes in all the parent graphs. Finally, the algorithm returns the identified common subgraphs and calculated similarity values (line 17). If a similarity value is greater than 0.8 for a particular subgraph, we identify that the subgraph and its parent call graphs satisfy Heuristic 1 .

The subgraphs which are common to all the call graphs in $\operatorname{SESE}(S)$ satisfy Heuristic 2. However, further processing is required to identify functions that act upon single BOs. To accomplish this check, we present Algorithm 2. 


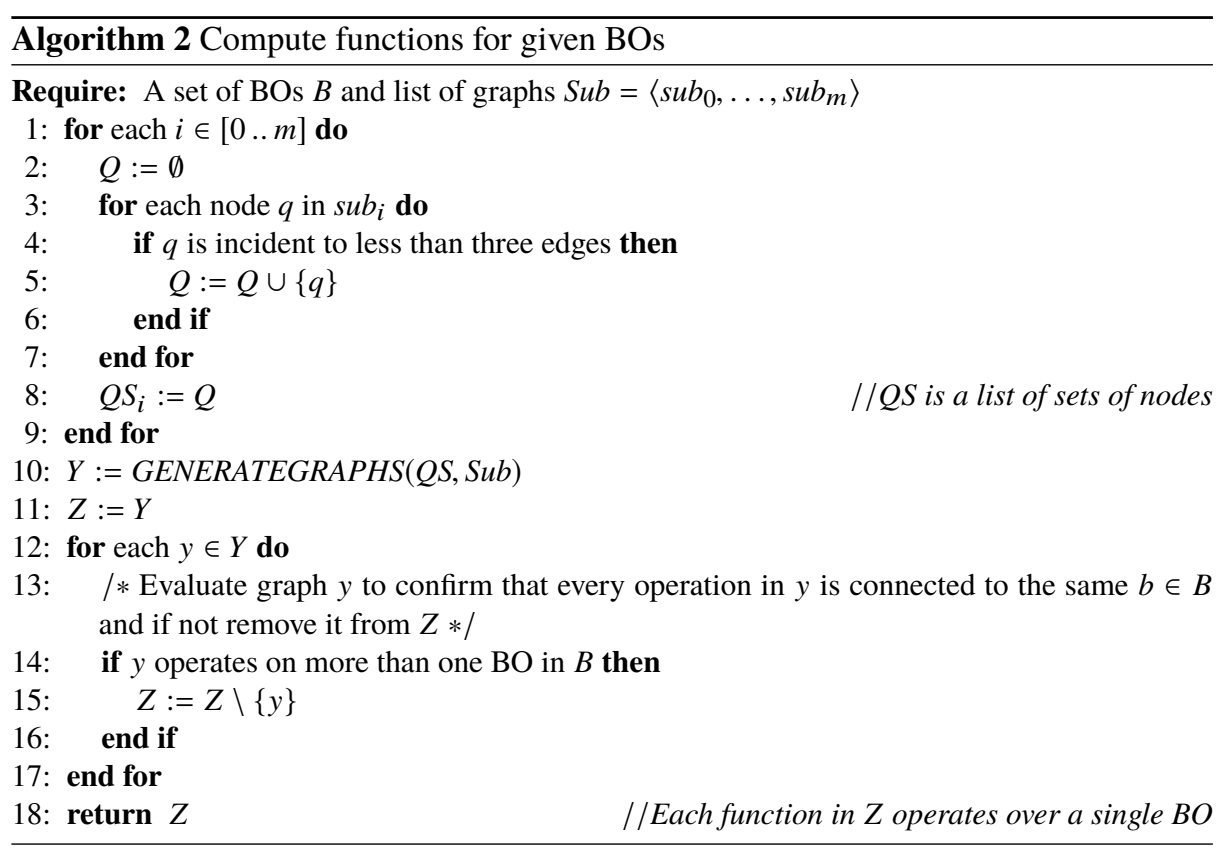

Algorithm 2 consists of three steps. The first step involves identifying the states of the input subgraphs with no more than two incident transitions, a.k.a single-entrysingle-exit states (lines 1-9). It is expected that the input subgraphs are generated by Algorithm 1. The loop of lines 1-9 iterates over all the subgraphs, while the loop of lines 3-7 runs over all the nodes of a current subgraph to extract and record the "SESE" states (line 5). The constructed sets of "SESE" states get stored in list QS on line 8.

In the second step of the algorithm function GENERATEGRAPHS constructs connected graphs composed of the nodes in $Q S$ that are subgraphs of the graphs in $S u b$, and records the result in set $Y$ (line 10).

\begin{tabular}{|c|c|c|c|c|c|c|c|c|}
\hline & $A$ & $B$ & $C$ & $D$ & $E$ & $F$ & $G$ & $H$ \\
\hline$A$ & 0 & 3 & 0 & 0 & 0 & 0 & 0 & 0 \\
\hline B & 0 & 0 & 2 & 3 & 0 & 0 & 0 & 0 \\
\hline C & 0 & 0 & 0 & 0 & 2 & 2 & 0 & 0 \\
\hline D & 0 & 0 & 0 & 0 & 0 & 3 & 0 & 0 \\
\hline E & 0 & 0 & 0 & 0 & 0 & 0 & 2 & 0 \\
\hline F & 0 & 0 & 0 & 0 & 0 & 0 & 3 & 0 \\
\hline G & 0 & 0 & 0 & 0 & 0 & 0 & 0 & 1 \\
\hline H & 0 & 0 & 0 & 0 & 0 & 0 & 0 & 0 \\
\hline
\end{tabular}

(a) AAM

\begin{tabular}{|c|c|c|c|c|c|c|c|c|}
\hline & $\mathrm{A}$ & $\mathrm{B}$ & $\mathrm{C}$ & $\mathrm{D}$ & $\mathrm{E}$ & $\mathrm{F}$ & $\mathrm{G}$ & $\mathrm{H}$ \\
\hline $\mathrm{A}$ & $\varnothing$ & $1,2,3$ & $\varnothing$ & $\varnothing$ & $\varnothing$ & $\varnothing$ & $\varnothing$ & $\varnothing$ \\
\hline $\mathrm{B}$ & $\varnothing$ & $\varnothing$ & 1,2 & $1,2,3$ & $\varnothing$ & $\varnothing$ & $\varnothing$ & $\varnothing$ \\
\hline $\mathrm{C}$ & $\varnothing$ & $\varnothing$ & $\varnothing$ & 0 & 1,2 & 1,2 & $\varnothing$ & $\varnothing$ \\
\hline $\mathrm{D}$ & $\varnothing$ & $\varnothing$ & $\varnothing$ & $\varnothing$ & $\varnothing$ & $1,2,3$ & $\varnothing$ & $\varnothing$ \\
\hline $\mathrm{E}$ & $\varnothing$ & $\varnothing$ & $\varnothing$ & $\varnothing$ & $\varnothing$ & $\varnothing$ & 1,2 & $\varnothing$ \\
\hline $\mathrm{F}$ & $\varnothing$ & $\varnothing$ & $\varnothing$ & $\varnothing$ & $\varnothing$ & $\varnothing$ & $1,2,3$ & $\varnothing$ \\
\hline $\mathrm{G}$ & $\varnothing$ & $\varnothing$ & $\varnothing$ & $\varnothing$ & $\varnothing$ & $\varnothing$ & $\varnothing$ & 1 \\
\hline $\mathrm{H}$ & $\varnothing$ & $\varnothing$ & $\varnothing$ & $\varnothing$ & $\varnothing$ & $\varnothing$ & $\varnothing$ & $\varnothing$ \\
\hline
\end{tabular}

(b) AGM

Fig. 4: Intermediate matrices used by Algorithm 1 computed for the call graphs in Fig. 2. 
In the third step, the algorithm evaluates each graph $y \in Y$ to verify whether all the operations captured in $y$ are carried out on the same BO (lines 11-17). If the operations relate to more than one $\mathrm{BO}$, the graph gets removed from set $Z$, which initially is assigned to contain all the graphs in $Y$. The BO mapping is achieved by evaluating each database table $t$ associated with operations of graph $y$ and mapping $t$ to the BOs that are characterized by $t$. If an operation, or several operations, of $y$ relates to database tables that characterize more than one BO, then $y$ gets removed from $Z$. At the end of the third step of Algorithm 2, set $Z$ is composed of all the functions that operate on a single BO, and this set is returned on line 18. Finally, the functions in set $Z$ get recommended to the user as possible MSs.

\section{Implementation and Validation}

A proper MS should provide high execution efficiency with a desirable level of scalability and availability. Furthermore the packages and components in it should be highly cohesive and loosely coupled $[1,16]$. In order to validate our MS discovery and recommendation process provides MSs with the desirable characteristics, we developed a prototype ${ }^{6}$ based on the algorithm presented in Section 3.2 and experimented on SugarCRM $^{7}$ and ChurchCRM ${ }^{8}$ which is detailed in our technical report [17].

This section only presents the details of the experiments that we conducted using the prototype on SugarCRM, which is a customer relationship management system that has a complex system structure with more than 8,000 source files, 600 attributes divided between 101 tables. We specifically focused on the campaign management module of SugarCRM to generate the execution sequences for our microservice discovery process. In order to cover all the user cases related to the campaign management module, 10 different executions ${ }^{9}$ related to the campaign management, such as target creation, campaign creation, and template creation, were performed and their log data was generated using the SugarCRM system's log functionality. The logs were then analyzed using the process mining tool Disco ${ }^{10}$ and 10 different call graphs were generated, all together containing around 200 unique execution nodes. The generated call graphs and the database tables were provided as the input to the prototype.

Discovered MSs: Based on the call graphs and database tables, the prototype identified three subtypes of campaigns, namely newsletter, email, and non-email, which results in functional splitting of the ES based on object subtypes (satisfying Heuristic 1). In addition, the prototype identified common sequences related to all the executions resulting in functional splitting of the ES based on execution fragments (satisfying Heuristic 2).

Validation Process: The validation process was conducted by implementing the recommended MSs in Google Cloud. Each MS was hosted in Google Cloud using a cluster of size 2 which has two virtual CPUs and a total memory of $7.5 \mathrm{~GB}$. The hosted MSs were exposed through the Google Cloud kubernetes API, allowing third

\footnotetext{
${ }^{6} \mathrm{https}: / /$ github.com/AnuruddhaDeAlwis/Subtype.git

7 https://www.sugarcrm.com/

8 http://churchcrm.io/

${ }^{9} \mathrm{http} / / /$ support.sugarcrm.com/Documentation/Sugar_Versions/8.0/Pro/Application_Guide/

$10 \mathrm{https} / / /$ fluxicon.com/disco/
} 
party computers to access them via API calls. In order to validate the sub-typing recommendations, we implemented three MSs simulating newsletter, email, and nonemail campaigns, and another system to simulate the legacy campaign module which covered all the campaign sub-types. In addition, we implemented a MS with common segments, i.e., fragments with similar states, communicating with other MSs simulating the common subgraphs recommendations given by the framework. Each MS was tested against a load of 150,000 requests and 300,000 requests generated by 10 machines simultaneously, simulating the customer requests, while recording their total execution time, average memory consumption and average disk consumption. The results are shown in Tables 1 and 2.

Based on the results reported in Tables 1 and 2, we calculated the scalability, availability, and execution efficiency of different combinations and the results obtained are summarized in Tables 3 and 4. The scalability was calculated according to the resources usage over time as described by Tsai et al. [13]. In order to determine the availability, first we calculated the time taken to process 100 requests if a particular MS is not available. Then, we used the difference between the total up-time and total down-time as described by Bauer et al. [14]. Efficiency gain was calculated by dividing the time taken by the legacy system to process all requests by the time taken by each MS. Furthermore we calculated the structural cohesion and coupling of the packages in legacy system and the new MS systems as described by Candela et al. [4].

Experimental Results: According to Tsai et al. [13], the lower the number the better the scalability. Thus, the newsletter and non-email MSs have better scalability than the legacy system when considering both memory and disk usage over time (refer to Table 3). In the email MS there is a scalability gain, even thought it is not as significant as that of the gain in the newsletter and non-email MSs. When considering availability we clearly observe that there is higher availability in subtype MSs than in the legacy system. As the number of requests increased from 150,000 to 300,000, subtype MSs were able to handle the request overload while providing better availability than the legacy system. Most importantly, when examining the request processing efficiency, each subtyping MS managed to process the request at at-least 1.5 times the speed of the legacy system.

Table 4 reports that there is not much of a gain in scalability and availability in the MS discovered and developed based on Heuristic 2 when compared with the legacy system. In contrast, when comparing the efficiency gain, it is evident that the common MS managed to process requests at at-least twice the accelerated speed of the legacy system. Furthermore, when comparing the coupling and cohesion values detailed in Table 5, it is evident that both campaign and common MSs attained a higher level of cohesion than the legacy system. In addition, the campaign MS managed to achieve slightly better coupling when compared with the legacy system even though there is a small increase in coupling in the common MS. Similar results were obtained for the experiments conducted on ChurchCRM's service management module [17].

Provided Solutions: The obtained results have affirmed that MSs extracted based on the recommendation of our prototype can provide the same services to the users while preserving overall system behaviour and achieving higher scalability, availability, efficiency, high cohesion, and low coupling. 


\begin{tabular}{|l|c|c|c|c|}
\hline Campaign Type & No. of Requests & Ex. Time (ms) & Avg Mem (GB) & Avg Disk (GB) \\
\hline Legacy & 150,000 & 324,000 & 3.00375 & 2.09550 \\
Legacy & 300,000 & 741,600 & 3.04025 & 2.10050 \\
Newsletter & 150,000 & 201,600 & 2.95475 & 2.09150 \\
Newsletter & 300,000 & 396,000 & 3.00575 & 2.09975 \\
Email & 150,000 & 198,000 & 2.89075 & 2.09225 \\
Email & 300,000 & 446,400 & 2.97075 & 2.10125 \\
Non-Email & 150,000 & 226,800 & 2.84550 & 2.09300 \\
Non-Email & 300,000 & 432,000 & 2.92875 & 2.10125 \\
\hline
\end{tabular}

Table 1: Legacy system vs subtype MSs execution results.

\begin{tabular}{|l|c|c|c|c|}
\hline System Type & No. of Requests & Ex. Time (ms) & Avg Mem (GB) & Avg Disk (GB) \\
\hline Legacy & 150,000 & 399,600 & 3.0335 & 2.0915 \\
Legacy & 300,000 & 781,200 & 3.1665 & 2.1020 \\
Common Seg. & 150,000 & 194,400 & 2.9110 & 2.0926 \\
Common Seg. & 300,000 & 396,000 & 2.9905 & 2.1015 \\
\hline
\end{tabular}

Table 2: Legacy system vs common subgraphs MSs execution results.

\begin{tabular}{|l|c|c|c|c|c|c|}
\hline $\begin{array}{l}\text { Campaign } \\
\text { Type }\end{array}$ & $\begin{array}{c}\text { Scalability } \\
{[\mathrm{Mem}]}\end{array}$ & $\begin{array}{c}\text { Scalability } \\
{[\text { Disk] }}\end{array}$ & $\begin{array}{c}\text { Availability } \\
{[150,000]}\end{array}$ & $\begin{array}{c}\text { Availability } \\
{[300,000]}\end{array}$ & $\begin{array}{c}\text { Efficiency } \\
{[150,000]}\end{array}$ & $\begin{array}{c}\text { Efficiency } \\
{[300,000]}\end{array}$ \\
\hline Legacy & 2.652 & 2.626 & 99.856 & 99.918 & 1.000 & 1.000 \\
Newsletter & 1.963 & 1.937 & 99.910 & 99.956 & 1.607 & 1.873 \\
Email & 2.612 & 2.552 & 99.912 & 99.950 & 1.636 & 1.661 \\
Non-Email & 1.867 & 1.821 & 99.899 & 99.952 & 1.429 & 1.717 \\
\hline
\end{tabular}

Table 3: Scalability, availability, and efficiency gains using subtyping.

\begin{tabular}{|l|c|c|c|c|c|c|}
\hline Campaign Type & $\begin{array}{c}\text { Scalability } \\
{[\mathrm{Mem}]}\end{array}$ & $\begin{array}{c}\text { Scalability } \\
{[\mathrm{Disk}]}\end{array}$ & $\begin{array}{c}\text { Availability } \\
{[150,000]}\end{array}$ & $\begin{array}{c}\text { Availability } \\
{[300,000]}\end{array}$ & $\begin{array}{c}\text { Efficiency } \\
{[150,000]}\end{array}$ & $\begin{array}{c}\text { Efficiency } \\
{[300,000]}\end{array}$ \\
\hline \hline Legacy & 1.9947 & 1.9205 & 99.9334 & 99.9667 & 1.0000 & 1.0000 \\
Common MS & 2.1314 & 2.0839 & 99.9334 & 99.9667 & 2.0556 & 1.9727 \\
\hline
\end{tabular}

Table 4: Scalability, availability, and efficiency gains using common subgraphs.

\begin{tabular}{|l|c|c|}
\hline System Type & Lack of Cohesion & Structural Coupling \\
\hline \hline Legacy with campaign packages & 104.00 & 17.00 \\
MS with campaign packages & 92.00 & 15.89 \\
Legacy with commonality packages & 55.83 & 18.00 \\
MS with commonality packages & 50.67 & 18.50 \\
\hline
\end{tabular}

Table 5: Comparison of lack of cohesion and structural coupling. 


\section{Related Work}

Microservices have emerged as the latest style of service-based software allowing systems to be distributed through the cloud as fine-grained components, typically with individual operations, in contrast to services under SOA which include all logically related operations [1]. Even though microservices can support the evolution of ERP systems by providing exploitation in cloud-enabled platforms such as the IoT [2], the research conducted in this particular area is limited. To the best of our knowledge there is no research related to the automation of MS discovery in legacy systems, apart from the manual migrations achieved by Balalaie et al. [15]. Balalaie et al. have described the complexity associated with the system reengineering process while pointing out the importance of considering BOs and their relationships in the migration system process. Martin Fowler emphasizes the importance of adapting BO relationships in microservices [16] aligning with the Domain Driven Design principles.

However, the existing software re-engineering techniques do not consider the complex relationship of BOs with their behaviours in the re-engineering process. Furthermore, studies show that the success rate of existing software re-modularisation techniques, especially for large systems, remains low [4]. A key stumbling block is the limited insights available from syntactic structures of software code for profiling software dependencies and evaluating their measurements for coupling and cohesion metrics [5]. As such, to derive successful re-engineering techniques, a methodology should consider the enriched semantic insights available through the BOs and functions in an ES.

In such a process, the first challenge would be identifying the BOs which are distributed among several database tables in an ES system, and identifying the relationships between them. Nooijen et al. [12] and Lu et al. [8] proposed methodologies and heuristics to identify BOs based on the database schema and information in database tables. However, according to Lu et al., the derived BOs might not be perfect and they have to be reclustered with the help of human expertise. A proper identification of BO relationships should consider the behavioural aspects of the systems as described by Hull [11]. However, there is still a gap in the area of correlating such behaviour with the underline BOs. As such, it is important to establish novel methodologies which incorporate both system behaviours and the business objects in the software re-engineering process.

\section{Conclusion}

This paper presented two heuristics used for functional splitting of ESs based on object subtypes and common execution fragments, while providing ground rules for MS discovery. A prototype was developed based on the proposed heuristics and validation was conducted by implementing the MSs recommended by the prototype for SugarCRM and ChurchCRM. The study has demonstrated that analysis of functions and BO CRUD operations while evaluating BO relationships helps to identify efficient solutions to migrate legacy systems into MSs with high cohesion and low coupling while achieving better scalability, availability, and execution efficiency. However, further analysis of BO relationships, such as inclusive and exclusive containment should be considered to further optimize the MS discovery process, and this will be carried out as future work. 


\section{References}

1. Newman, S., 2015. Building MSs NGINX. O'Reilly.

2. Columbus L., (2017). 2017 Internet Of Things (IoT) Intelligence Update. Available from https://www.forbes.com/sites/louiscolumbus/2017/11/12/2017-internet-of-thingsiot-intelligence-update/43aa6f4c7f31

3. Magal, S.R. and Word, J., 2011. Integrated business processes with ERP systems. Wiley Publishing.

4. Candela, I., Bavota, G., Russo, B. and Oliveto, R., 2016. Using cohesion and coupling for software remodularization: Is it enough?. ACM Transactions on Software Engineering and Methodology (TOSEM), 25(3), p.24.

5. Anquetil, N. and Laval, J., 2011, March. Legacy software restructuring: Analyzing a concrete case. In Software Maintenance and Reengineering (CSMR), 2011 15th European Conference on (pp. 279-286). IEEE.

6. Barros, A., Decker, G., Dumas, M., and Weber, F. (2007). Correlation patterns in serviceoriented architectures. Proceedings of the $10^{t h}$ International Conference on Fundamental Approaches to Software Engineering (FASE), pages 245 - 259. Springer.

7. Pẽrez Castillo, R., Garcĩa Rodríguez de Guzmãn, I., Caballero, I. and Piattini, M., 2013. Software modernization by recovering Web services from legacy databases. Journal of Software: Evolution and Process, 25(5), pp.507-533.

8. Lu, X., Nagelkerke, M., van de Wiel, D. and Fahland, D., 2015. Discovering interacting artifacts from ERP systems. IEEE Transactions on Services Computing, 8(6), pp.861-873.

9. Polyvyanyy, A., Vanhatalo, J. and Volzer, H., 2010, September. Simplified computation and generalization of the refined process structure tree. In International Workshop on Web Services and Formal Methods (pp. 25-41). Springer, Berlin, Heidelberg.

10. Halpin, T. and Morgan, T., 2010. Information modeling and relational databases. Morgan Kaufmann.

11. Hull, R., 2008. Artifact-centric business process models: Brief survey of research results and challenges. In On the Move to Meaningful Internet Systems (pp. 1152-1163). Springer

12. Nooijen, E.H.J, van Dongen, B. F. and Fahland, D., 2012. Automatic discovery of data-centric and artifact-centric processes. In International Conference on Business Process Management (pp. 316-327). Springer

13. Tsai, W.T., Huang, Y. and Shao, Q., 2011, December. Testing the scalability of SaaS applications. In Service-Oriented Computing and Applications (SOCA), 2011 IEEE International Conference on (pp. 1-4). IEEE.

14. Bauer, E. and Adams, R., 2012. Reliability and availability of cloud computing. John Wiley \& Sons.

15. Balalaie, A., Heydarnoori, A. and Jamshidi, P., 2015, September. Migrating to cloud-native architectures using MSs: an experience report. In European Conference on Service-Oriented and Cloud Computing (pp. 201-215). Springer, Cham.

16. Fowler, M. (2014). Microservices a definition of this new architectural term. Avaialble from: https://martinfowler.com/articles/microservices.html

17. De Alwis, A., Barros, A., Polyvyanyy, A., and Fidge, C. “Technical Report: Function-Splitting Heuristics for Discovery of Microservices in Enterprise Systems" 2018. [Online]. Available: https://eprints.qut.edu.au/119030/ 